\title{
GUT MICROBIOTA CHANGE AND TIME OF RESTORE IN INTENSIVE CARE THERAPY: A CASE REPORT
}

\author{
Francesca Borgo ${ }^{1}$, Elisa Borghi ${ }^{1}$, Silvia Marzorati ${ }^{3}$, Gaetano lapichino ${ }^{2,3}$, Giulia Morace ${ }^{1}$
}

\section{ARTICLE INFO \\ Received: $14^{\text {th }}$ Oct 2015 \\ Revised: $30^{\text {th }}$ Nov 2015 \\ Accepted: $3^{\text {rd }}$ Dec 2015}

Author details: ${ }^{1}$ Department of Health Sciences, Università degli Studi di Milano, via di Rudinì, 8, Milan, Italy; ${ }^{2}$ Department Pathophysiology and Transplantation, Università degli Studi di Milano, via F. Sforza, 35 , Milan, Italy, ${ }^{3}$ Intensive Care Unit, San Paolo Hospital, via di Rudinì, 8, Milan, Italy

Correspondig Author: Giulia Morace, Department of Health Sciences, Università degli Studi di Milano, via di Rudinì, 8, Milan, Italy

Email: giulia.morace@unimi.it

\section{INTRODUCTION}

Exposure of gut microbiota to antibiotics selects for multidrug-resistant organisms, which can be a major clinical problem. Antimicrobial treatment not only target pathogenic bacteria, but also can have damaging effects on the ecology of commensal species [1]. The longer is the exposure to drugs, such as in critically ill patients, the deeper is the impact on microbial ecology, eventually leading to a complete ecological collapse. The selective pressures imposed by modern intensive care therapy, including multiple antibiotic exposure, provision of all nutrients exclusively through intravenous route (total parenteral nutrition) is the main driving force of microbial changes. However, the gut microbiota seems to be relatively resilient to antibiotic challenge, and after shortterm modification returns to its pre-treatment composition [2]. A better understanding of the ecological perturbations that characterize the microbiota during prolonged intensive care therapy, in terms of both composition and restoration time, is crucial to develop new supportive strategies in intensive care patients. Here we characterized the biodiversity and composition of gut microbial communities (bacterial and fungal) in a patient hospitalized in intensive care unit.

\section{CASE REPORT}

A 54 years old male patient with no previous medical history was admitted in September 2014 to the Emergency Department of San Paolo Hospital in Milan with a diagnosis of acute cholecystitis with significant biliary duct leakage. The same day, he underwent urgent surgery and was admitted at the Intensive Care Unit (ICU) due to a septic syndrome. SOFA and SAPS

score were 9 and 37, respectively, with a predicted mortality risk of $18 \%$.

During ICU stay, the patient underwent several surgical and non-surgical procedures: nasobiliary and transhepathic percutaneous drainage (PTBD); endoscopic retrograde cholangiopancreatography (ERCP) and biliary duct endoprothesis positioning; open abdomen treatment in severe compartment syndrome. ICU hospitalization lasted 51 days. The patient suffered of a septic shock with a hemodynamic instability that required invasive monitoring and vasopressors, leucopenia (WBC 2300), high procalcitonin levels (PCT). At day 8, blood and bile culture gave back Escherichia coli and E. coli/ Klebsiella pneumoniae, respectively. A combined antibiotic therapy was started (Table 1).

Table 1 Antibiotic therapy

\begin{tabular}{llll}
\hline $\begin{array}{l}\text { Antibiotic } \\
\text { therapy }\end{array}$ & Dosage & $\begin{array}{l}\text { Administra } \\
\text { tion route }\end{array}$ & $\begin{array}{l}\text { Period } \\
\text { (from day- } \\
\text { to day) }\end{array}$ \\
\hline Cefazolin & $6 \mathrm{~g} / \mathrm{die}$ & iv $^{*}$ & $1-4$ \\
Metronidazole & $2 \mathrm{~g} / \mathrm{die}$ & iv & $1-6$ \\
Fluconazole & $200 \mathrm{mg} / \mathrm{die}$ & $\mathrm{ngt}^{\S}$ & $3-17$ \\
Cefotaxime & $12 \mathrm{~g} / \mathrm{die}$ & iv & $4-8$ \\
Gentamicin & $560 \mathrm{mg} / \mathrm{die}$ & iv & $5-12$ \\
\hline
\end{tabular}




\begin{tabular}{llll}
\hline Meropenem & $4 \mathrm{~g} / \mathrm{die}$ & iv & $12-17$ \\
Colistin & $4000000 \mathrm{UI}$ & ngt & $13-18$
\end{tabular}

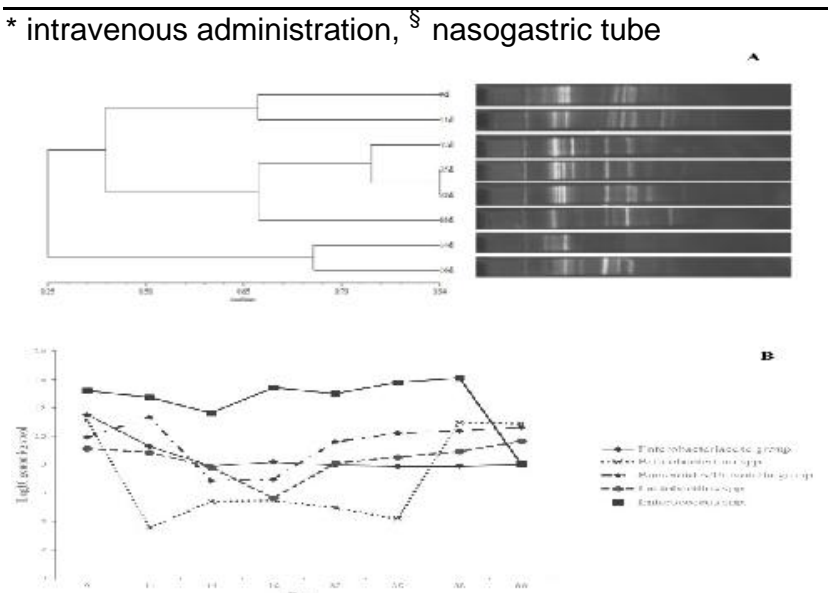

Fig1. Fecal Gut Microbiota Ecology. A The UPGMA dendrogram obtained for the DGGE analysis; B Quantification of microrganisms detected in fecal samples using real time PCR. Results are expressed in Log10 of genome number/g stool.

At day 9, K. pneumoniae was also isolated from blood culture and E.coli in bronchial tube. At day 11, bile culture was positive for Proteus spp., E. coli, Enterococcus faecium and $E$. faecalis. At day 20, a progressive resolution and recovery of organs function were observed, together with respiratory weaning till spontaneous breathing. The antibiotic therapy was then discontinued. Nutritional support has been guaranteed via enteral feeding (Nutrison ST: $500 \mathrm{kcal}$ 1st day, 1000 kcal 2nd day; Prosure (Abbot) $1500 \mathrm{Kcal} /$ day). Spontaneous oral feeding started at day 40 , with a consequent reduction in enteral nutritional support.

No feces were passed until day 8; from day 8 till 13 diarrhea developed (1000-2500 ml/die), then fecal transit normalized (200-300 ml/die).

Fecal samples were collected and immediately stored at $-80^{\circ} \mathrm{C}$ until analysis, at days $9,11,14,16,32,35,38$ and at day 88, after strong amelioration of patient's clinical conditions and admission to the Internal Medicine ward. Total bacterial DNA extractions were performed with $200 \mathrm{mg}$ fecal material using the Spin stool DNA kit (Stratec Molecular, Berlin, Germany), according to the manufacturer's instructions. PCR amplifications of total bacterial DNA were obtained using the primer pair HDA1GC and HDA2, and the thermocycling program as described previously [4]. The Denaturing Gradient Gel Electrophoresis (DGGE) analysis was performed with a Dgene System (Bio-Rad Hercules) using a $0.8 \mathrm{~mm} 8 \%$ polyacrylamide gel with $35-60 \%$ gradient of urea and formamide, as reported previously [5]. Calculation of similarity in the profiles of bands was based on Dice correlation coefficient (Figure 1). Dendrogram was obtained by means of the Un- weighted Pair Group Method using Arithmetic Average (UPGMA) clustering algorithm.

Presence and quantification of the most representative species and microbial groups (referred as core microbiota) was carried out by Real-time PCR quantification (qPCR, Figure 2). qPCR was carried out using the StepOne instrument (Applied Biosystems), the SYBR® Green chemistry (ThermoScientific, USA). Standard curves were obtained for each qPCR run using serial dilutions of control DNA. The specific 16S rRNA primers and QPCR conditions used for Bacteroides/Prevotella group, Bifidobacterium spp. And Enterobacteriaceae group are reported by Bartosch and colleagues [6]; for Lactobacillus spp. We used the conditions designed by Delroisse et al. [7].

\section{DISCUSSION}

In this case report, we monitored patient's gut microbiota during ICU hospitalization and eventually at his recovery from critical conditions. Different reports are available on the effects of artificial nutrition and antibiotic treatment on gut microbial diversity. Artificial nutrition promotes the reduction of fecal volume and gut microbiota dysbiosis that eventually could be implicated in a leaky gut condition [8]. The dysbiosis is worsened by antibiotics administration, and could selects resistant microorganisms that overgrowth and colonize the gastrointestinal tract of patients. Prolonged and broadspectrum therapies are associated to deeper changes in the microbial

ecology [6].

Due to the critical conditions of the patient, we used several combinations of antimicrobial therapies, driven by isolation of different pathogens during the ICU staying. As expected, deep changes in the microbial ecology accompanied the use of supportive nutrition and antibiotics.

DGGE profiles of all collected samples set up two main clusters (46\% similarity by Dice correlation index): one represented by samples at days 14 and $16(77 \%$ of similarity), the other cluster included 2 sub-clusters $(65 \%$ similarity), days 9 and 11, and days 32, 35, 38 and 88 (Figure 1A).

The previous patient history did not report any inflammatory gut disease. Day 9 sample could be representative of the initial situation, being the first stool that has been passed since hospital admission. Although a diarrhea condition developed, we observed the most dramatic change after 14 days of antibiotic treatment. Day 14 and 16 samples showed the lower degree in microbial biodiversity and corresponded to the simultaneous Fluconazole, Meropenem, Colistin treatment (Table 1).

It is interesting to note that samples from days 32,35 and 38 clustered at $80 \%$ of similarity. These samples where obtained during patient's ICU staying after antibiotics discontinuation and normal feeding initiation. The last sample, collected during the patient convalescence in Internal Medicine ward (day 88, without antibiotic treatment and normal alimentation), presented a DGGE profile with a $65 \%$ of similarity with days 32,35 and 38 . We confirmed DGGE results by absolute quantification of the most representative bacterial groups (Figure 1B). 
Despite day 9 was the first stool the patient has passed since hospital admission, quantification of bacterial species and genera showed a higher abundance of Enterococcus spp. and Enterobacteriaceae compared with healthy population [9]. The antibiotic treatment started at the admission in ICU ward, thus it could have influenced the overgrowth of these species, according to literature data [7]. E. faecalis, a species with high antibiotic resistance traits, as well as E.coli and $P$. mirabilis ESBL (extended spectrum beta-lactamase)producing were cultured form bile at day 11 . We cannot exclude their translocation from gut.

From day 11 till day 16 we observe a dramatic decrease in Bifidobacterium spp., Lactobacillus spp., Bacteroides spp. ; then, these core microbiota genera started to gradually regrow from day 32 to day 88,16 days after antimicrobial therapy discontinuation.

The abundance in Enterococcus spp. and Enterobacteriaceae remained above normal until the patient's discharge from ICU: the decrease in their gut content concurred with normalization of other gut commensals.

Bifidobacterium spp. took longer to stabilize, starting to regrow at day 35 , and reaching a normal abundance at day 38. This genus might exert an inhibitory activity against Enterobacteriaceae and Enterococcus spp., by producing acetate and lowering the intestinal $\mathrm{pH}[10]$.

\section{CONCLUSION}

The case report here presented shows that short period of antimicrobial therapy could trigger a remarkable dysbiosis, in terms of both biodiversity and concentrations. However, the gut microbiota is a resilient entity with a species-specific recovery time. The delay in the regrow of some species, i.e. Bifidobacterium spp., could promote transient colonization with potentially pathogenic species that can overgrow and eventually translocate causing systemic infection.

Probiotic administration of key species during antimicrobial therapy could thus favor the reduction of dysbiosis and promote a more rapid return to a healthy microbial community

\section{REFERENCES}

1. Cotter PD, Stanton C, Ross RP, Hill C. The impact of antibiotics on the gut microbiota as revealed by high throughput DNA sequencing. Discov Med. 2012;13:193-99.

2. De La Cochetière MF, Durand $T$, Lepage $P$, Bourreille A, Galmiche JP, Doré J. Resilience of the dominant human fecal microbiota upon short-course antibiotic challenge. J Clin Microbiol. 2005;43:558892.

3. Le Gall JR, Loirat P, Alperovitch A, Glaser P, Granthil C, Mathieu D, et al. A simplified acute physiology score for ICU patients. Crit Care Med. 1984;12:975-7.

4. Walter J, Tannock GW, Tilsala-Timisjarvi A, Rodtong $S$, Loach DM, Munro $K$ et al. Detection and identification of gastrointestinal Lactobacillus species by using denaturing gradient gel electrophoresis and species-specific PCR primers. Appl Environ Microbiol. 2000;66,297-03.

5. Iapichino G, Callegari ML, Marzorati S, Cigada M, Corbella D, Ferrari $S$, et al. Impact of antibiotics on the gut microbiota of critically ill patients. J Med Microbiol. 2008;57:1007-14.

6. Bartosch S, Fite A, Macfarlane GT, McMurdo MET. Characterization of bacterial communities in feces from healthy elderly volunteers and hospitalized elderly patients by using Real-Time PCR and effects of antibiotic treatment on the fecal microbiota. Appl Environ Microbiol.2004;6,3575-81.

7. Delroisse JM, Bolvin AL, Parmentier I, Dauphin RD, Vandenbol M, Portetelle D. Quantification of Bifidobacterium spp. and Lactobacillus spp. in rat fecal samples by real-time PCR. Microbiol Res.2008;163,663-70.

8. Schneider SM, Raina C, Pugliese P, Pouget I, Rampal P, Hébuterne X. Outcome of patients treated with home enteral nutrition. JPEN J Parenter Enteral Nutr. 2001; 25:203-09.

9. Malinen E, Rinttilä T, Kajander K, Mättö J, Kassinen A, Krogius L, et al. Analysis of the fecal microbiota of irritable bowel syndrome patients and healthy controls with real-time PCR. Am J Gastroenterol. 2005;100:373-82.

10. Umenai $T$, Shime $N$, Asahara $T$, Nomoto $K$, Itoi T. A pilot study of Bifidobacterium breve in neonates undergoing surgery for congenital heart disease. J Intensive Care. 2014;2:36. 